\title{
An Attack on Two Hash Functions by Zheng-Matsumoto-Imai
}

\author{
Bart Preneel, René Govaerts, and Joos Vandewalle \\ Katholieke Universiteit Leuven, Laboratorium ESAT-COSIC, \\ Kardinaal Mercierlaan 94, B-3001 Heverlee, Belgium
}

\begin{abstract}
In [ZMI89,ZMI90] two constructions for a collision resistant hash function were proposed. The first scheme is based on a block cipher, and the second scheme uses modular arithmetic. It is shown in this paper that both proposals have serious weaknesses.
\end{abstract}

\section{Introduction}

For an informal definition of a collision resistant hash function the reader is referred to [PGV92]. The following model will be used to described iterated hash functions:

$$
H_{i}=f\left(X_{i}, H_{i-1}\right) \quad i=1,2, \ldots t .
$$

Here $f$ is the round function, $X_{i}$ are the $t$ message blocks, $H_{i}$ are the chaining variables, $H_{0}$ is equal to the initial value, that should be specified together with the scheme, and $H_{t}$ is the hashcode. It was shown by I. Damgård [Dam89] that if the round function $f$ is a collision resistant function, $h$ is a collision resistant hash function. The authors of [ZMI89,ZMI90] claim that their constructions yield a collision resistant round function. It will be demonstrated that in both cases the round function is not collision resistant, and that in some cases collisions for $h$ can be constructed.

\section{The Hash Function Based on a Block Cipher}

The round function $f$ compresses a 224-bit input to a 128-bit output and is based on $\mathrm{xDES}^{1}$. This block cipher is one of the extensions of DES [Fi46] that has been proposed in [ZMI89b]. xDES ${ }^{1}$ is a three round Feistel cipher with block length 128 bits, key size 168 bits and with the $F$ function equal to DES. One round is defined as follows:

$$
C 1_{i+1}=C 2_{i} \quad \text { and } \quad C 2_{i+1}=C 1_{i} \oplus \operatorname{DES}\left(K_{i}, C 2_{i}\right) \quad i=0,1,2 .
$$

The variables $C 1_{i}$ and $C 2_{i}$ are 64 -bit blocks, and $K_{i}$ are 56-bit keys. The block cipher is then written as

$$
C 2_{3} \| C 1_{3}=\operatorname{xDES}^{1}\left(K_{1}\left\|K_{2}\right\| K_{3}, C 1_{0} \| C 2_{0}\right) .
$$

\footnotetext{
* NFWO aspirant navorser, sponsored by the National Fund for Scientific Research (Belgium).
}

Appeared in Advances in Cryptology - ASIACRYPT 1992, Lecture Notes in Computer Science 718, J. Seberry, and Y. Zheng (eds.), Springer-Verlag, pp. 535-538.

(c)1992 Springer-Verlag 
Here $C 1_{0}$ and $C 2_{0}$ are the first and second part of the plaintext, and $C 2_{3}$ and $C 1_{3}$ are the first and second part of the ciphertext. The collision resistant function consists of $2 \mathrm{xDES}^{1}$ operations:

$$
f(Y 1 \| Y 2)=\operatorname{xDES}^{1}\left(\operatorname{chop}_{72}\left(\operatorname{xDES}^{1}(\beta \| Y 1, \alpha)\right) \| Y 2, \alpha\right) .
$$

Here $Y 1$ and $Y 2$ are 112-bit blocks, $\alpha$ is a 128 -bit constant, $\beta$ is a 56-bit initialization variable and chop $_{r}$ drops the $r$ least significant (or rightmost) bits of its argument. The complete hash function has the following form: $H_{i}=f\left(H_{i-1} \| X_{i}\right)$, where $H_{i-1}$ is a 128 -bit block, and $X_{i}$ is a 96 -bit block. The rate of this scheme is equal to 4 , which means that 4 DES encryptions are required to hash 64 bits.

The scheme has two weaknesses, that allow to produce collisions for the round function $f$. First only 56 bits are kept from the first xDES ${ }^{1}$ encryption, and hence a birthday attack will require only $2^{29}$ operations to produce a collision for the intermediate value and hence for the function $f$. The second problem is that if $\beta=K_{1}$ and $Y_{1}=K_{2} \| K_{3}$, one can use the key collision search algorithm described in [QD89] to produce key collisions for the DES plaintext equal to the second part of $\alpha$. This yields a collision for $f$ in about $2^{33}$ operations.

The scheme can be strengthened however by distributing $\beta$ equally over $K_{1}$, $K_{2}$, and $K_{3}$, and by increasing the size of $\beta$ [Zhe92]. It will be shown that independently of the size of $\beta$, the security level can not be larger than 44 bits. If the size of $\beta$ is equal to $v$ bits (in the original proposal $v=56$ ), the number of fixed bits of $\beta$ that enter the key port of a single DES block is equal to $v / 3$ (it will be assumed that $v$ is divisible by 3 ). It can be shown that the rate of this scheme is then equal to $R=\frac{6 \cdot 64}{208-2 v}$. The number of bits of $Y_{1}$ that enter the key port will be denoted with $y$, hence $y+v / 3=56$. Two attacks are now considered.

For the fixed value of the right part of $\alpha$ and of the first $v / 3$ bits of $\beta$, one can calculate and store a set of $2^{z}$ different ciphertexts. The probability that a collision will be found in this set is approximately equal to $2^{2 z-65}$. If $y>32$, implying $v<72$, a value of $z=33$ is clearly sufficient to obtain a collision. If on the other hand $y \leq 32$, one will take $z=y$, and the probability of success is smaller than one. One can however repeat this procedure, (e.g., if one attacks a DES block different from the first one, a different value can be chosen for the value of the bits of $Y_{1}$ that enter the first DES), and the expected number of operations for a single collision is equal to $2^{65-y}$, while the required storage is equal to $2^{y}$. An extension of the Quisquater algorithm [QD89] could be used to eliminate the storage. If the security level $S$ is expressed in bits, it follows that $S=\max \{65-y, 33\}$. With the relation between $y$ and $v$, one obtains $S=\max \{9+v / 3,33\}$.

A second attack follows from the observation that only $v$ bits are kept from the output of the first $\mathrm{xDES}^{1}$ operation (hence the chop operation is chopping $128-v$ bits). It is clear that finding a collision for the remaining $v$ bits requires only $2^{v / 2+1}$ operations, or $S \leq v / 2+1$ bits. This attack is more efficient than the first attack if $v<64$ bits.

The relation between $S$ and $v$ can be summarized as follows: if $v<64$ then $S=v / 2+1$, if $64 \leq v<72$ then $\mathrm{S}=33$, and if $72 \leq v<104$ then $S=v / 3+9$.

Appeared in Advances in Cryptology - ASIACRYPT 1992, Lecture Notes in Computer Science 718, J. Seberry, and Y. Zheng (eds.), Springer-Verlag, pp. 535-538.

(c)1992 Springer-Verlag 
One can conclude that producing a collision for the proposed round function requires less than $2^{44}$ operations. Depending on the allocation of the bits of $X_{i}$ and $H_{i-1}$ to $Y_{1}$ and $Y_{2}$, it might also be feasible to produce a collision for the hash function with a fixed initial value: it is certainly possible to produce a collision for the hash function if there is a single DES block where all key bits are selected from $X_{i}$.

\section{The Hash Function Based on Modular Arithmetic}

In this case the round function $f$ consists of 2 modular squarings with an $n$-bit modulus (with $n=500$ ):

$$
f(Y 1 \| Y 2)=\left(\operatorname{chop}_{450}\left((\beta \| Y 1)^{2} \bmod N\right) \| Y 2\right)^{2} \bmod N
$$

where chop' ${ }_{r}(x)$ drops the $r$ most significant bits of $x, Y 1$ and $Y 2$ are 450-bit blocks, and $\beta$ is a 50 -bit initialization variable. The complete hash function has the following form: $H_{i}=f\left(H_{i-1} \| X_{i}\right)$, where $H_{i-1}$ is a 500-bit block, and $X_{i}$ is a 400-bit block. The security of this scheme is based on the fact that $O(\log N)$ bits of squaring modulo $N$ is hard if $N$ is a Blum integer, i.e., $N=p q$ with $p \equiv q \equiv 3 \bmod 4$. From this it is wrongly concluded that finding two integers such that their squares agree at the 50 least significant positions is hard (a trivial collision for $x$ is $x^{\prime}=-x$ ). As only 50 bits of the first squaring are used as input to the second squaring, it follows that collisions can be found with a birthday attack in $2^{26}$ operations. It can be shown that one can find a second preimage and hence a collision for $f$ even if $k=n / 4$ bits are selected, or $3 n / 4$ bits are chopped. The algorithm is the same as the one presented in [Gir87] to break a related scheme with redundancy in the least significant positions.

\section{References}

[Dam89] I.B. Damgård, "A design principle for hash functions," Advances in Cryptology, Proc. Crypto'89, LNCS 435, G. Brassard, Ed., Springer-Verlag, 1990, pp. $416-427$.

[Fi46] "Data Encryption Standard," Federal Information Processing Standard (FIPS), Publication 46, National Bureau of Standards, U.S. Department of Commerce, Washington D.C., January 1977.

[Gir87] M. Girault, "Hash-functions using modulo-n operations," Advances in Cryptology, Proc. Eurocrypt'87, LNCS 304, D. Chaum and W.L. Price, Eds., Springer-Verlag, 1988, pp. 217-226.

[PGV92] B. Preneel, R. Govaerts, and J. Vandewalle, "On the power of memory in the design of collision resistant hash functions," these proceedings.

[QD89] J.-J. Quisquater and J.-P. Delescaille, "How easy is collision search ? Application to DES," Advances in Cryptology, Proc. Eurocrypt'89, LNCS 434, J.-J. Quisquater and J. Vandewalle, Eds., Springer-Verlag, 1990, pp. 429-434.

[ZMI89] Y. Zheng, T. Matsumoto, and H. Imai, "Duality between two cryptographic primitives," Papers of technical group for information security, IEICE of Japan, March 16, 1989, pp. 47-57.

Appeared in Advances in Cryptology - ASIACRYPT 1992, Lecture Notes in Computer Science 718, J. Seberry, and Y. Zheng (eds.), Springer-Verlag, pp. 535-538.

(C)1992 Springer-Verlag 
[ZMI89b] Y. Zheng, T. Matsumoto, and H. Imai, "On the construction of block ciphers provably secure and not relying on any unproved hypothesis," Advances in Cryptology, Proc. Crypto'89, LNCS 435, G. Brassard, Ed., Springer-Verlag, 1990, pp. 461-480.

[ZMI90] Y. Zheng, T. Matsumoto, and H. Imai, "Duality between two cryptographic primitives," Proc. 8th International Conference on Applied Algebra, Algebraic Algorithms and Error-Correcting Codes, LNCS 508, S. Sakata, Ed., SpringerVerlag, 1991, pp. 379-390.

[Zhe92] Y. Zheng, personal communication, 1992.

Appeared in Advances in Cryptology - ASIACRYPT 1992, Lecture Notes in Computer Science 718, J. Seberry, and Y. Zheng (eds.), Springer-Verlag, pp. 535-538. (C)1992 Springer-Verlag 